Rev. Elev. Méd. vét. Pays trop., 1978, 31 (1) : 85-90.

\title{
Influence d'une alimentation intensive sur les performances de reproduction des femelles zébus Gobra au CRZ de Dahra
}

\author{
par J. P. DENIS $\left({ }^{*}\right)$ et A. I. THIONGANE $\left({ }^{* *}\right)$
}

\begin{abstract}
RÉSUMÉ
La distribution à volonté d'un concentré alimentaire équilibré à des femelles zébu Gobra depuis leur naissance a considérablement avancé l'âge de leur premier vêlage par rapport à ce qui est normalement constaté dans le troupeau d'élevage traditionnel.

Cette amélioration dans l'alimentation des femelles en observation a par contre faiblement influencé l'espacement des vêlages et la production lactée.

Les auteurs voient dans cette méthode le moyen le plus efficace pour lutter contre le manque de précocité des femelles Gobra dans la reproduction et de ce fait valoriser l'exploitation du troupeau.
\end{abstract}

\section{INTRODUCTION}

En 1968, une expérience visant à extérioriser les potentialités du zébu Gobra a été démarrée au Centre de Recherches Zootechniques de Dahra. Les travaux ont tout d'abord porté sur les possibilités de croissance maximale d'animaux des 2 sexes pris au hasard dans le troupeau du Centre au fur et à mesure de leur naissance.

On s'est proposé ensuite de mesurer l'influence de l'alimentation intensive distribuée sur les performances de reproduction des femelles. En effet, le nombre de veaux produits durant la vie de reproduction est un facteur primordial de productivité, surtout chez les femelles à vocation bouchère.

L'intervalle entre les vêlages calculé chez les animaux Gobra du Centre de Recherches Zoo-

(*) Adresse actuelle : I. E. M. V. T. 10, rue PierreCurie, 94700 Maisons-Alfort.

(**) Laboratoire National de l'Elevage et de Recherches Vétérinaires, B. P. 2057, Dakar-Hann, République du Sénégal. techniques de Dahra-Djoloff est de 473 jours en moyenne, soit 15 mois environ. A l'extérieur de la station, les résultats, tels qu'ils ressortent d'enquêtes approfondies effectuées depuis plusieurs années, montrent que cet intervalle est de 20 mois et plus, ce qui signe un taux de fécondité très faible ( 60 p. 100 et moins).

L'influence de l'alimentation a paru forte sur ces phénomènes : ce qui nous a amené à conduire cette expérience dont les résultats font l'objet du présent document.

\section{MATÉRIEL}

\section{Animaux expérimentaux}

Il s'agit de 28 femelles Gobra choisies au fur et à mesure de leur naissance à partir du mois d'avril jusqu'au mois de septembre 1968. Ces femelles sont issues de reproductrices à plusieurs niveaux de sélection et de vie de reproduction (primi ou multipares). 


\section{Animaux témoins}

Les lots d'animaux témoins sont constitués de toutes les femelles nées au C. R. Z. en 1965-66 et 67 pour ce qui concerne l'étude de l'évolution pondérale et staturale.

Pour l'étude des performances de reproduc. tion, les femelles témoins sont des femelles d'âge équivalent non supplémentées.

\section{MÉTHODES}

\section{MODE D'ENTRETIEN DES ANIMAUX}

\section{Abreuvement}

Pour tous les animaux, l'abreuvement est à volonté dans des abreuvoirs mis à leur disposition dans tous les parcs.

\section{Alimentation}

Le protocole alimentaire appliqué aux animaux apparaît au tableau $\mathrm{n}^{\circ} \mathrm{I}$.

Dans tous les cas le pâturage naturel constitue r'alimentation de base.

Ce pâturage est essentiellement composé de graminées annuelles précoces fines comme Digitaria, Aristida, Chloris, Eragrostis..., grossières ou tardives telles Diheteropogon, Andropogon, Ctenium... et de légumineuses comme Tephrosia, Indigofera, Zornia. En fait, les proportions relatives des différentes espèces sont très variables suivant en cela l'influence déterminante de la pluviométrie.

La composition des concentrés 1 et 2 successivement distribués aux animaux expérimentaux est la suivante (tabl. $\mathrm{n}^{\circ} \mathrm{II}$ ) :

TABL. $N^{\circ}$ II-Composition des deux concentrës distribués successivement aux lots d'extériorisation

\begin{tabular}{|c|c|c|}
\hline & $N^{d} I$ & $N^{\circ} 2$ \\
\hline Niais & 10 & 10 \\
\hline Son de blé & 38 & 40 \\
\hline Son de maĩs & 16 & 17 \\
\hline Son de sorgho & 28 & 29,50 \\
\hline Tourteau d'ardchide & 5,25 & 0,50 \\
\hline Carbonzte de Ca & 2 & 2,25 \\
\hline Chlorure de sadium & 0,5 & 0,50 \\
\hline Complément vi taminé & 0,25 & 0,25 \\
\hline
\end{tabular}

\section{Habitat}

Les femelles ont été élevées dans des parcelles de pâturage extensif. Les aliments étaient placés dans des mangeoires, sous un abri sommaire qui pouvait cependant apporter suffisamment d'ombre aux animaux pendant les heures chaudes de la journée.

\section{VIE DE REPRODUCTION}

Les femelles ont été placées à la reproduction selon le protocole suivant (tabl. $n^{\circ}$ III) avec le taureau Gobra $\mathrm{n}^{\circ} 2400$. Les animaux du lot extériorisé et du lot témoin ont été respectivement partagés en 2 lots.

TABL, $\mathbb{N}^{\circ} \mathrm{I}-\mathrm{A}$ limentation des différents lots

\begin{tabular}{|c|c|c|}
\hline & Ex tê $r i$ or i, s ês & T é moins \\
\hline Naissance au sevrage & $\begin{array}{l}\text { Lait maternel, concentré } \\
n^{\circ} 1 \text { a volonte }\end{array}$ & $\begin{array}{l}\text { Lait maternel concentré } \mathrm{D}^{0} 3 \\
(0,80 \mathrm{UF}-90 \mathrm{~g} \mathrm{MAD} / \mathrm{kg} 500 \mathrm{~g} / \mathrm{j}\end{array}$ \\
\hline Sevrage à 12 mois & $\begin{array}{l}\text { Pâturage nature } 1 \text {, concentré } \\
n^{\circ} 1 \text { à volonté }\end{array}$ & $\begin{array}{l}\text { Pâturage naturel, concentrê } \\
\mathrm{n}^{\circ} 3 \mathrm{l} \mathrm{kg} / \mathrm{j} / \text { tête. }\end{array}$ \\
\hline 12 mois à 30 mois & $"$ & Pâturage nature 1 \\
\hline 30 mois à 43 mois & $\begin{array}{l}\text { Pâturage naturel, concentré } \\
\text { n* } 2 \text { à volonté }\end{array}$ & " \\
\hline 43 mois à 66 mois & $\begin{array}{l}\text { Pâturage naturel, concentré } \\
\mathrm{n}^{\circ} 210 \mathrm{~kg} / \mathrm{j} / \text { tête }\end{array}$ & $"$ \\
\hline$>66$ mois & $\begin{array}{l}\text { Pâturage nature } 1 \text {, concentrê } \\
\mathrm{n}^{\circ} 25 \mathrm{~kg} / \mathrm{j} / \text { tête. }\end{array}$ & $n$ \\
\hline
\end{tabular}


TABLEAU N"III

\begin{tabular}{|l|c|c|c|}
\hline \multirow{2}{*}{ Extèriorisès } & Lots & Janvier & Août \\
\cline { 2 - 4 } & $\mathbf{I}$ & $\mathbf{x}$ & $\mathbf{x}$ \\
\cline { 2 - 4 } & III & & $\mathrm{x}$ \\
\hline \multirow{2}{*}{ Témoins } & II & $\mathbf{x}$ & $\mathbf{x}$ \\
\cline { 2 - 4 } & IV & & $\mathrm{x}$ \\
\hline
\end{tabular}

En fait, ce protocole a été involontairement modifié. En effet, 6 femelles du lot III (extériorisé) ont été saillies avant que le lot ne soit placé à la reproduction. Elles se trouvaient à proximité des jeunes taurillons d'extériorisation dont certains ont franchi les clôtures et les ont fécondées. Les 6 autres ont été fécondées au mois d'août au moment de leur mise à la reproduction conformément au protocole expérimental. Le même incident s'est produit pour une des femelles du lot témoin IV fécondée en janvier.

Quelques incidents ont marqué la vie des différents lots pendant la période d'étude : réformes (fractures), pathologie (toxi-infections), dystocies... mais la diminution du nombre des animaux en observation a été répartie de façon équilibrée entre les différents lots, ce qui n'a par conséquent pas influencé de manière notable les résultats.

\section{RÉSULTATS, DISCUSSION}

\section{Age au 1er vêlage (tabl. $n^{\circ}$ IV)}

On constate que par rapport à la moyenne générale du troupeau de départ, les animaux témoins gagnent 5 mois environ, ce qui peut être imputé aux meilleures conditions d'entretien et à la sélection pratiquée. En ce qui concerne les femelles extériorisées, le gain est de 9 mois supplémentaires, soit en tout 14 mois environ. Cette fois, le gain obtenu peut être imputé à l'action de l'alimentation distribuée et il est très significatif $(F=54,2)$.

\section{Temps passé entre la mise à la reproduction et la fécondation}

Le tableau $n^{\circ} \mathrm{V}$ montre le temps passé entre la mise à la reproduction et la $1^{\text {re }}$ fécondation pour les 4 lots. La durée de la gestation est comptée 10 mois ( $293 \pm 4$ jours).

Dans le lot I, 91 p. 100 des animaux ont été fécondés au cours des 3 premiers mois. Une femelle ne l'a été qu'après 10 mois de présence avec le mâle.

Dans le lot III, 5 femelles sur 6 ont été fécondées immédiatement, 1 durant le $4^{e}$ mois $\mathrm{d}$

TABLEAU N*IV

\begin{tabular}{|c|c|c|c|}
\hline & $\begin{array}{l}\text { Troupeau tout venant } \\
\qquad(1955-1970)\end{array}$ & T É moins & Extêriorisês \\
\hline $\begin{array}{c}\text { Age au } \\
\text { ler vêlage }\end{array}$ & $\begin{array}{c}1365 \pm 24 \text { jours } \\
45 \text { mois } \\
(n: 534)\end{array}$ & $\begin{array}{c}1184 \pm 55 \text { jours } \\
40 \text { mois } \\
(n=24)\end{array}$ & $\begin{array}{c}933 \pm 46 \text { jours } \\
31 \text { mois } \\
(n=24)\end{array}$ \\
\hline
\end{tabular}

TABL. $N^{\circ} \mathrm{V}$-Temps passê entre la mise au taureau et la féconciation pour les quatre lots.

\begin{tabular}{|c|c|c|c|c|c|c|c|c|c|c|c|c|c|c|c|c|c|c|c|}
\hline Mois & \multicolumn{12}{|c|}{$A n \cap \overrightarrow{\mathrm{e}}$ e $\mathrm{I}$} & \multicolumn{7}{|c|}{ A n n é e II } \\
\hline Lots & 1. & 2 & 3 & 4 & 5 & 6 & 7 & 8 & 9 & 10 & 11 & 12 & 13 & 14 & 15 & 16 & 17 & 18 & 19 \\
\hline I & 8 & 2 & 1 & & & & & & & 1 & & & & & & & & & \\
\hline \multirow{2}{*}{ III } & 5 & & & 1 & & & & & & & & & & & & & & & \\
\hline & & & & & & & & 3 & 3 & & & & & & & & & & \\
\hline II & & 2 & & 1 & & 3 & 1 & 1 & & 1 & I & & & & & & & 1 & 1 \\
\hline IV & 1 & & & & & & & 7 & 1 & 2 & 1 & & & & & & & & \\
\hline & & & & & \multicolumn{3}{|c|}{ Mois } & 1 & 2 & 3 & 4 & 5 & 6 & 7 & 8 & 9 & 10 & 11 & 12 \\
\hline
\end{tabular}


présence. Pour les femelles placées à la reproduction en août, les 6 fécondations ont été observées au cours des 2 premiers mois.

Dans le lot II, 83 p. 100 des femelles sont fécondées après 11 mois de présence avec le taureau. Les fécondations sont échelonnées durant cette période. Deux femelles ne sont fécondées qu'au cours des $18^{\circ}$ et $19^{\circ}$ mois de présence.

Dans le lot IV, toutes les femelles ont été fécondées durant les 4 premiers mois de présence (sauf une, fécondée par accident dès janvier).

Il faut noter que pour les lots témoins II et IV, la date de la mise à la reproduction (18 et 25 mois) n'a pas influencé de façon importante les dates limites de fécondation. Mais en moyenne, les femelles mises plus tôt à la reproduction ont un âge au $1^{\text {er }}$ vêlage inférieur d'une façon significative $(1101 \pm 59 \mathrm{j}$. contre $1262 \pm 66 \mathrm{j} \mathrm{F}=15$ ) de 5 mois environ. Au moment où le lot IV est placé à la reproduction (août), les femelles du lot II ont été fécondées à 58,5 p. 100 ;

En conclusion, il ressort de cette discussion qu'il y a intérêt à placer les femelles le plus tôt possible à la reproduction, même si leur régime n'est pas très amélioré, pour gagner du temps au niveau de la vie de reproduction du troupeau par fécondation rapide des femelles les plus précoces.

Cette mesure reste valable si l'on examine les intervalles entre les vêlages dans les 2 lots témoins, qui sont significativement identiques.

Quant aux femelles supplémentées, il est évident qu'on peut les placer très tôt à la reproduction.

\section{Intervalles entre Ies vêlages}

Les résultats apparaissent au tableau $n^{\circ}$ VI.

TABL. $N^{\circ}$ VI-Intervalles entre les' vêtlages.

\begin{tabular}{|l|c|c|}
\hline Lots & $1-2$ & $2-3$ \\
\hline Extériorisés & $384,4 \pm 25,3 \mathrm{j}$ & $371,5 \pm 44,1 \mathrm{j}$ \\
\hline Ténoins & $419,7 \pm 40,2 \mathrm{j}$ & $398,4 \pm 41,2 \mathrm{j}$ \\
\hline $\mathrm{F}$ & $2,37 \mathrm{NS}$ & $0,86 \mathrm{NS}$ \\
\hline
\end{tabular}

On constate qu'il existe une différence moyenne de 1 mois entre les lots supplémentés et témoins. Cette différence n'est pas significative, ce qui est peut-être dû au faible nombre d'animaux observés.

\section{Nombre de veaux en fonction de l'âge des femelles}

En fait, en conclusion, la notion finalement essentielle que l'on doit retirer de cette expérience est le nombre de veaux obtenus dans les différents lots.

L'expérience a été stoppée au $3^{\text {e }}$ vêlage pour l'ensemble des femelles. Le calcul porte donc sur l'âge moyen des femelles au moment du $3^{e}$ vêlage (tabl. $n^{\circ}$ VII).

TABL. $\mathbb{N}^{\circ}$ VII-Age moyen des femelles au $3 e$ vêlage

\begin{tabular}{|c|c|c|}
\hline \multirow[b]{2}{*}{ Extëriorisës } & \multicolumn{2}{|c|}{ Age au $3 \mathrm{e}$ vêlage } \\
\hline & $1663,84 \pm 42,8 j$ & 4 ans $1 / 2$ \\
\hline Témoins & $1977,84+61,67 j$ & 5 ans $1 / 2$ \\
\hline $\mathbf{F}$ & 76,36 & - \\
\hline
\end{tabular}

En fait, les données concernant les deux lots témoins sont significativement différentes. L'écart de 5 mois environ qui existait au moment du premier vêlage se retrouve au $3^{\mathrm{e}}$ vêlage: (lot II : $1888 \pm 67$; Lot IV : $2058 \pm 73$; $\left.\mathrm{F}=14,46\left({ }^{*}\right)\right)$.

En conclusion, au $3^{\mathrm{e}}$ vêlage, les femelles supplémentées sont plus jeunes de 314 jours, soit 10 mois. Le fait d'appliquer une supplémentation permet, par conséquent, de prévoir raisonnablement 1 ou 2 veaux supplémentaires durant la vie reproductive de la femelle. Le gain principal se situant au niveau de l'âge au premier vêlage.

\section{Répartition mensuelle des naissances}

Dans les conditions naturelles d'exploitation du troupeau, la majorité des naissances a lieu durant une certaine période de l'année : soit pendant les mois de juin à septembre. Pour expliquer cette fréquence importante des saillies fécondes à une période particulière de l'année, le facteur alimentaire avait été avancé comme responsable essentiel.

L'expérience d'extériorisation agissant sur le facteur alimentaire paraît confirmer cette vue. 


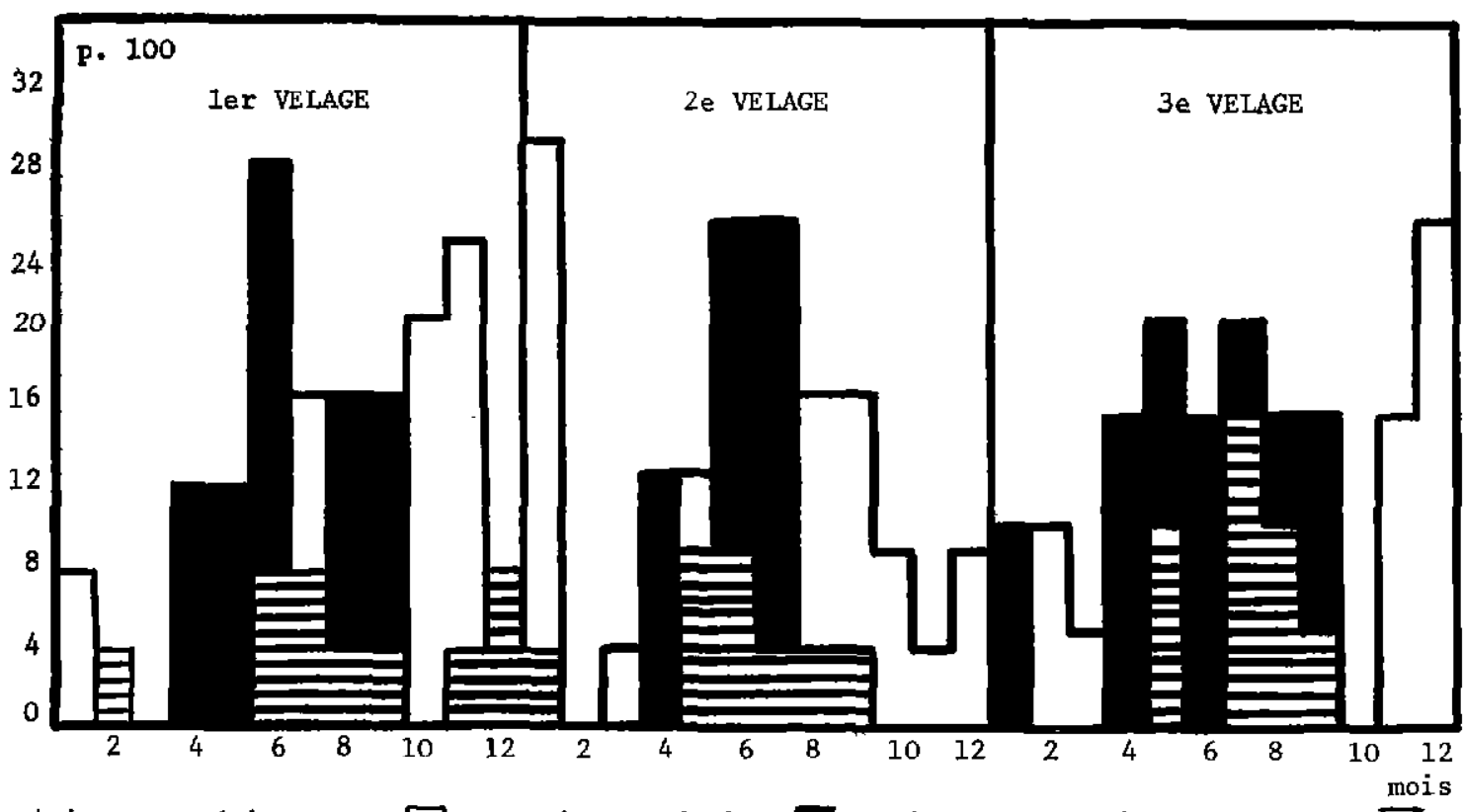

Animaux expérimentaux

Animaux témoins

Fréquence conjointe des 2 lots

\section{Taux de gémellarité}

La gémellarité est très peu fréquente chez le zébu Gobra. Sur 2711 naissances enregistrées de 1954 à 1972, seulement 7 naissances gemellaires ont été observées (soit un taux de 0,25 p. 100).

Par contre, chez les femelles d'extériorisation, on note pour 66 naissances (vêlages 1 à 3 ), 2 naissances gémellaires, soit un taux de 3 p. 100 .

Par conséquent, au niveau de la fertilité, l'influence de l'alimentation paraît se faire sentir d'une manière importante.

\section{INFLUENCE SUR LES QUALITÉS LAITIËRES}

Cet aspect de l'expérience a été peu exploré en raison des difficultés de la traite chez ces femelles. Néanmoins, des quelques contrôles effectués, il résulte que les valeurs des lactations obtenues sont extrêmement faibles $(328 \mathrm{~kg}$ en 130 jours $=2,5 \mathrm{l} / \mathrm{j}$ ) et confirment bien les faibles qualités laitières de ces animaux, leur production n'étant pratiquement pas augmentée par l'extériorisation.

Le contrôle indirect par l'étude de la croissance des produits n'a pu être entrepris, puisque ceux-ci recevaient la même alimentation distribuée à volonté jusqu'à 18 mois pour les abattages à 12 et 18 mois. 


\section{CONCLUSION}

L'influence de l'alimentation sur la vie de reproduction des femelles Gobra est très grande. On peut en conclure que le manque de précocité observé chez ces femelles est essentiellement dû à l'insuffisance de la ration alimentaire dont elles souffrent depuis leur naissance. Un élevage rationnel, en particulier sur le plan alimentaire, permet d'espérer l'augmentation très nette du nombre des veaux viables d'une part et précoces d'autre part et donc d'augmenter d'une manière concrète la productivité du troupeau.

\section{SUMMARY}

Influence of intensive feeding on reproductive performances of Gobra zebu cows at the Dahra CRZ

The ad libitum balanced concentrate supply to Gobra zebu cows since birth has considerably hastened the first calving time in comparison with the average figures in the traditional breeding herd.

However, this feeding improvement of the females had almost no influence on calving spacing and dairy production.

The authors conclude that this method is the most efficient way to palliate the lack of reproductive prococity of Gobra zebu cows and therefore to improve herd performances.

\section{RESUMEN}

Influencia de una alimentación intensiva sobre los rendimientos de la reproducción de hembras cebu Gobra en el Centro de investigaciones zootécnicas de Dahra

La distribución ad libitum de un concentrado alimenticio equilibrado a vacas cebú Gobra desde su nacimiento ha adelantado considerablemente la edad de su primer parto en relación con la encontrada en animales de ganađeria tradicional. En cambio, esta mejora de la alimentación de las hembras ha tenido poca influencia sobre la duración entre los partos y la producción de leche. Según los autores, dicho método es el medio más eficaz para luchar contra la falta de precocidad de la reproducción en las hembras Gobra y pués para valorizar la explotación del ganado.

\section{BIBLIOGRAPHIE}

1. COSTIOU (P.). Rapport d'enquête sur le cheptel bovin du Ferlo. Dakar, I. E. M. V. T., Laboratoire National de l'Elevage, novembre 1972,40 p.

2. DENIS (J. P.). Note sur l'âge au premier vêlage chez le zébu Gobra. Communication à la III ${ }^{\mathrm{e}}$ Conférence Internationale de Zootechnie. Versailles, juillet 1971.

3. DENIS (J. P.). L'intervalle entre les vêlages chez le zébu Gobra. Rev. Elev. Méd. vét. Pays trop., 1971, $24(4): 635-647$.

4. DENIS (J. P.). Influence des facteurs bioclimatiques sur la reproduction des femelles zébu en milieu tropical sec. Communication au VII ${ }^{e}$ Congrès de Repro- duction Animale et d'Insémination artificielle, Munich 6-9 juin 1972.

5. DENIS (J. P.). Note sur les facteurs conduisant au choix d'une saison de monte au C. R. Z, de Dabra (Sénégal). Rev. Elev. Méd. vét. Pays trop., 1975, 28 (4) : 491-97.

6. DENIS (J. P.), VALENZA (J.). Etude et sélection du zébu Peulh sénégalais (Gobra). Communication au Congrès Mondial de Production Animale. Université de Maryland, U. S. A., juillet 1968.

7. FAYOLLE (F.). Rapport d'enquête sur le cheptel bovin. Région d'Amaly. Dakar, I. E. M. V. T. Laboratoire National de l'Elevage, août 1972, 24 p. 